TITLE:

\title{
Entropy production from chaoticity in Yang-Mills field theory with use of the Husimi function
}

\section{$\operatorname{AUTHOR}(\mathrm{S}):$}

Tsukiji, Hidekazu; lida, Hideaki; Kunihiro, Teiji; Ohnishi, Akira; Takahashi, Toru T.

\section{CITATION:}

Tsukiji, Hidekazu ... [et al]. Entropy production from chaoticity in Yang-Mills field theory with use of the Husimi function. Physical Review D 2016, 94(9): 091502(R).

\section{ISSUE DATE:}

2016-11-01

URL:

http://hdl.handle.net/2433/233194

RIGHT:

(C) 2016 American Physical Society. 
PHYSICAL REVIEW D 94, 091502(R) (2016)

\title{
Entropy production from chaoticity in Yang-Mills field theory with use of the Husimi function
}

\author{
Hidekazu Tsukiji, ${ }^{1, *}$ Hideaki Iida, ${ }^{2, \dagger}$ Teiji Kunihiro, ${ }^{2}$ Akira Ohnishi, ${ }^{1}$ and Toru T. Takahashi ${ }^{3}$ \\ ${ }^{1}$ Yukawa Institute for Theoretical Physics, Kyoto University, Kyoto 606-8502, Japan \\ ${ }^{2}$ Department of Physics, Faculty of Science, Kyoto University, Kyoto 606-8502, Japan \\ ${ }^{3}$ Gunma National College of Technology, Gunma 371-8530, Japan
}

(Received 28 March 2016; published 23 November 2016)

\begin{abstract}
We investigate possible entropy production in Yang-Mills (YM) field theory by using a quantum distribution function called the Husimi function $f_{\mathrm{H}}(A, E, t)$ for the YM field, which is given by a coarse graining of the Wigner function and non-negative. We calculate the Husimi-Wehrl entropy $S_{\mathrm{HW}}(t)=$ $-\operatorname{Tr} f_{\mathrm{H}} \log f_{\mathrm{H}}$ defined as an integral over the phase space, for which two adaptations of the test-particle method are used combined with Monte Carlo method. We utilize the semiclassical approximation to obtain the time evolution of the distribution functions of the YM field, which is known to show chaotic behavior in the classical limit. We also make a simplification of the multidimensional phase-space integrals by making a product ansatz for the Husimi function, which is found to give a 10-20\% overestimate of the HusimiWehrl entropy for a quantum system with a few degrees of freedom. We show that the quantum YM theory does exhibit the entropy production and that the entropy production rate agrees with the sum of positive Lyapunov exponents or the Kolmogorov-Sinai entropy, suggesting that the chaoticity of the classical YM field causes the entropy production in the quantum YM theory.
\end{abstract}

DOI: 10.1103/PhysRevD.94.091502

\section{INTRODUCTION}

Thermalization or entropy production in an isolated quantum system is a long-standing problem. The entropy of a quantum system may be given by von Neumann entropy $S_{\mathrm{vN}}=-\operatorname{Tr} \rho \log \rho$ with $\rho$ being the density matrix [1], and taking into account that the time evolution of the quantum system is described by a unitary transformation $U(t)=\mathrm{e}^{-i H t / \hbar}$, von Neumann entropy $S_{\mathrm{vN}}$ is shown to remain unchanged in time, which is an absurd consequence in contradiction to reality. One possible way to avoid this puzzle is to assume that there is no isolated quantum system because any quantum system is surrounded by the environment composed of quantum fields described by, say, QED; the partial trace with respect to the environment would lead to a density matrix of a mixed state due to the entanglement [2]. For thermalization of a macroscopic quantum system, the old idea of von Neumann was recently rediscovered, and since then a lot of related works and developments have been made [3,4]; see Ref. [5] and references cited therein. It might be worth mentioning that the entanglement entropy of a quantum system may have a geometrical interpretation as is clearly shown by Ryu-Takayanagi's formula [6].

In this work, we do not intend to develop a master theory to describe thermalization or entropy production of a generic quantum system. Instead, we concentrate on entropy production in quantum systems of which the classical

\footnotetext{
*tsukiji@yukawa.kyoto-u.ac.jp

Present address: Far Eastern National University, 690950 Vladivostok, Russia.
}

counterparts are chaotic and the semiclassical approximation is valid. There are many physical systems satisfying these characteristics [7]: among them, we have in mind the problem of the early thermaliztion in high-energy heavy-ion collisions (see the review [8] and recent studies [9-25]) at the Relativistic Heavy-Ion Collider in the Brookhaven National Laboratory [26-29] and the Large Hadron Collider at CERN [30].

Chaotic classical systems are characterized by the sensitive dependence of the trajectory on the initial condition, and trajectories starting from adjacent initial conditions with the difference $\delta X(0)$ in the phase space deviate exponentially $|\delta X(t)|=\exp (\lambda t)|\delta X(0)|$ from each other; the exponent $\lambda$ is called a Lyapunov exponent. Then, one can readily imagine that the chaotic behavior makes the phase-space distribution $f(q, p)$ so complicated that it generates a finite amount of entropy via a coarse graining in the classical Hamiltonian system. In this respect, it is interesting that the sum of positive Lyapunov exponents coincides with the Kolomogorov-Sinai entropy (see references in Ref. [31]) or the production rate of entropy [32]. Indeed, these have been demonstrated for a discrete classical system [33], where an explicit calculation of the Boltzmann-like entropy $S_{\mathrm{B}}=-\operatorname{Tr} f \log f$ was made with the distribution function $f(q, p)$ as obtained by a coarse graining of the phase space of the discrete system.

A natural extension of the above interesting work to a quantum systems might be done with the application of the quantum mechanical distribution function, i.e., the Wigner function $f_{\mathrm{W}}(q, p)$ derived as a Weyl transform of the density matrix $\rho$ [34]. However, since $f_{\mathrm{W}}$ is a mere Weyl transform of $\rho$, it cannot describe an entropy production of 
a pure quantum system, even apart from the fact that $f_{\mathrm{W}}$ is not positive definite.

To circumvent this well-known difficulty, let us recall that one cannot distinguish two phase-space points in a unit cell in quantum mechanics, and smearing in the phasespace volume of $(2 \pi \hbar)^{D}$ may be allowed, where $D$ is the degrees of freedom (DOF) of the system. For such a smeared distribution function, we adopt the Husimi function $f_{\mathrm{H}}$ [35], which is obtained by a Gaussian smearing of the Wigner function and is semipositive definite. Then, we can define the entropy in terms of $f_{\mathrm{H}}$ as $S_{\mathrm{HW}}=-\operatorname{Tr} f_{\mathrm{H}} \log f_{\mathrm{H}}$, where $\operatorname{Tr}$ means the integral over the phase space. This entropy was first introduced and called the classical entropy by Wehrl [36], and we call it Husimi-Wehrl (HW) entropy [37,38]. It should be noted that the HW entropy is a kind of microscopic entropy, and does not necessarily increase in time. Indeed, it may show an oscillatory behavior in time depending on the initial configuration as shown for quantum mechanical systems [38]; we will find that it is also the case for the Yang-Mills field. Nevertheless, it is noteworthy that the HW entropy in the thermal equilibrium tends to coincide with the von Neumann entropy at high temperatures [37].

In the previous work [38], the present authors examine thermalization of isolated quantum systems by using the HW entropy evaluated in the semiclassical approximation. It was shown that the semiclassical treatment works well in describing the entropy-production process of a couple of quantum mechanical systems of which the classical countersystems are known to be chaotic. Two novel methods were also proposed to evaluate the time evolution of the HW entropy, the test-particle method and the two-step Monte Carlo method, and it was demonstrated that the simultaneous application of the two methods ensures the reliability of the results of the HW entropy at a given time.

In this article, we extend the previous work [38] to the Yang-Mills (YM) field, which is known to be chaotic and has a macroscopic number of positive Lyapunov exponents [18]. We investigate the possible entropy production by constructing the Husimi function and calculating the HW entropy of the YM field in the semiclassical approximation. The initial condition we adopt for the equation of motion (EOM) of the YM field is motivated by the early stage of relativistic heavy-ion collisions [7,39].

There is, however, a caveat against this simple prescription that works for quantum mechanical systems with a few degrees of freedom because of the large number of the degrees of freedom in the field theory. Thus, we also take a simple ansatz for the Husimi function, where we construct it by a product of the Husimi function for each degree of freedom, although the classical EOM itself is solved numerically with the fully included nonlinear couplings. When applied to a quantum mechanical system with 2 degrees of freedom, the ansatz gives a $10-20 \%$ overestimate of the HW entropy. We also develop a novel efficient numerical method for calculating the HW entropy, which is a modification of the test-particle method. We calculate the HW entropy in YM field theory in a semiclassical way for the first time and show that the entropy production rate agrees with the sum of positive Lyapunov exponents or Kolmogorov-Sinai (KS) entropy.

\section{HUSIMI-WEHRL ENTROPY ON THE LATTICE}

We consider the $\mathrm{SU}\left(N_{c}\right)$ YM field on a $L^{3}$ lattice. In the temporal gauge, the Hamiltonian in noncompact formalism is given by

$$
H=\frac{1}{2} \sum_{x, a, i} E_{i}^{a}(x)^{2}+\frac{1}{4} \sum_{x, a, i, j} F_{i j}^{a}(x)^{2},
$$

with $F_{i j}^{a}=\partial_{i} A_{j}^{a}(x)-\partial_{j} A_{i}^{a}(x)+\sum_{b, c} f^{a b c} A_{i}^{b}(x) A_{j}^{c}(x) . N_{D}=$ $3 L^{3}\left(N_{c}^{2}-1\right)$ is the total DOF. We take the dimensionless gauge field $A$ and conjugate momentum $E$ normalized by the lattice spacing $a$ throughout this article. The coupling constant $g$ is also included in the definitions of $A$ and $E$.

The Husimi-Wehrl entropy of the YM field is obtained as a natural extension of that in quantum mechanics by regarding $(A(x), E(x))$ as canonical variables. First, we define the Wigner function (referred to as the Wigner functional [40]) in terms of $A(x)$ and $E(x)$,

$$
\begin{aligned}
f_{\mathrm{W}}[A, E ; t]= & \int \frac{D A^{\prime}}{(2 \pi \hbar)^{N_{D}}} e^{i E \cdot A^{\prime} / \hbar} \\
& \times\left\langle A+A^{\prime} / 2|\hat{\rho}(t)| A-A^{\prime} / 2\right\rangle,
\end{aligned}
$$

where $A \cdot E=\sum_{i, a, x} A_{i}^{a}(x) E_{i}^{a}(x)$ is the inner product. The time evolution of the Wigner function is derived from the von Neumann equation,

$$
\frac{\partial}{\partial t} f_{\mathrm{W}}[A, E ; t]=\frac{\partial H}{\partial A} \cdot \frac{\partial f_{\mathrm{W}}}{\partial E}-\frac{\partial H}{\partial E} \cdot \frac{\partial f_{\mathrm{W}}}{\partial A}+\mathcal{O}\left(\hbar^{2}\right) .
$$

In the semiclassical approximation, we ignore $\mathcal{O}\left(\hbar^{2}\right)$ terms, and then $f_{\mathrm{W}}$ is found to be constant along the trajectory satisfying the classical EOM (a good review can be found in Ref. [41]),

$$
\dot{E}=-\frac{\partial H}{\partial A}, \quad \dot{A}=\frac{\partial H}{\partial E} .
$$

Second, we introduce the Husimi function as the smeared Wigner function with the minimal Gaussian packet,

$$
\begin{aligned}
f_{\mathrm{H}}[A, E ; t]= & \int \frac{D A^{\prime} D E^{\prime}}{(\pi \hbar)^{N_{D}}} \mathrm{e}^{-\Delta\left(A-A^{\prime}\right)^{2} / \hbar-\left(E-E^{\prime}\right)^{2} / \Delta \hbar} \\
& \times f_{\mathrm{W}}\left[A^{\prime}, E^{\prime} ; t\right],
\end{aligned}
$$


where $\Delta$ is the parameter for the range of Gaussian smearing. As in quantum mechanics, the Husimi function is semi-positive definite; $f_{\mathrm{H}}[A, E ; t] \geq 0$, and we define the Husimi-Wehrl entropy as Boltzmann's entropy or Wehrl's classical entropy [36] by adopting the Husimi function for the phase-space distribution,

$$
S_{\mathrm{HW}}(t)=-\int \frac{D A D E}{(2 \pi \hbar)^{N_{D}}} f_{\mathrm{H}}[A, E ; t] \log f_{\mathrm{H}}[A, E ; t] .
$$

\section{NUMERICAL METHODS}

We calculate the time evolution of the HW entropy by two methods: the test particle (TP) method and the parallel test particle (pTP) method. The TP method is developed in Ref. [38]. The pTP method, an alternative method for the two-step Monte Carlo method, requires less numerical cost and gives almost the same results as the two-step Monte Carlo method. We have demonstrated that the HW entropy in some quantum mechanical systems is successfully obtained in these two methods, which are reviewed in the following.

In the test particle method (TP) for the Yang-Mills field theory, the "test particles" represent the gauge field amplitude $A$ and its canonical conjugate $E$, and in terms of these variables, the Wigner function is a sum of the delta functions,

$f_{\mathrm{W}}[A, E ; t]=\frac{(2 \pi \hbar)^{N_{D}}}{N_{\mathrm{TP}}} \sum_{i=1}^{N_{\mathrm{TP}}} \delta^{N_{D}}\left(A-A_{i}(t)\right) \delta^{N_{D}}\left(E-E_{i}(t)\right)$,

where $N_{\mathrm{TP}}$ is the total number of the test particles. The initial conditions of the test particles are $\left(A_{i}(0), E_{i}(0)\right)$ $\left(i=1,2, \ldots, N_{\mathrm{TP}}\right)$, which are chosen so as to sample well $f_{\mathrm{W}}[A, E, 0]$. The time evolution of the coordinates $\left(A_{i}(t), E_{i}(t)\right)$ is determined so that it reproduces the EOM for $f_{\mathrm{W}}[A, E, t]$, which is reduced to the canonical EOM (4) in the semiclassical approximation.

With the test particle representation of the Wigner function, Eq. (7), the Husimi function is readily expressed as

$$
f_{\mathrm{H}}[A, E ; t]=\frac{2^{N_{D}}}{N_{\mathrm{TP}}} \sum_{i=1}^{N_{\mathrm{TP}}} e^{-\Delta\left(A-A_{i}(t)\right)^{2} / \hbar-\left(E-E_{i}(t)\right)^{2} / \Delta \hbar} .
$$

It is noteworthy that the Husimi function here is a smooth function in contrast to the corresponding Wigner function in Eq. (7).

Substituting the Wigner function (8) into Eq. (6), the HW entropy in the test particle method is finally given as

$$
\begin{aligned}
S_{\mathrm{HW}}^{(\mathrm{TP}, \mathrm{pTP})} & =-\frac{1}{N_{\mathrm{TP}}} \sum_{i=1}^{N_{\mathrm{TP}}} \int \frac{D A D E}{(\pi \hbar)^{N_{D}}} e^{-\Delta\left(A-A_{i}(t)\right)^{2} / \hbar-\left(E-E_{i}(t)\right)^{2} / \Delta \hbar} \log \left[\frac{2^{N_{D}}}{N_{\mathrm{TP}}} \sum_{j=1}^{N_{\mathrm{TP}}} e^{-\Delta\left(A-A_{j}(t)\right)^{2} / \hbar-\left(E-E_{j}(t)\right)^{2} / \Delta \hbar}\right] \\
& \simeq-\frac{1}{N_{\mathrm{MC}} N_{\mathrm{TP}}} \sum_{k=1}^{N_{\mathrm{MC}}} \sum_{i=1}^{N_{\mathrm{TP}}} \log \left[\frac{2^{N_{\mathrm{D}}}}{N_{\mathrm{TP}}} \sum_{j=1}^{N_{\mathrm{TP}}} e^{-\Delta\left(\bar{A}_{k}+A_{i}(t)-A_{j}(t)\right)^{2} / \hbar-\left(\bar{E}_{k}+E_{i}(t)-E_{j}(t)\right)^{2} / \Delta \hbar}\right] .
\end{aligned}
$$

Note here that the integral over $(A, E)$ has a support only around the positions of the test particles $\left(A_{i}(t), E_{i}(t)\right)$ due to the Gaussian function for each $i$, and we can effectively perform the Monte Carlo integral. We generate random numbers $\left(\mathcal{A}_{i, k}, \mathcal{E}_{i, k}\right)\left(k=1, \ldots, N_{\mathrm{MC}}\right)$ with zero mean and standard deviations of $(\sqrt{\hbar / 2 \Delta}, \sqrt{\hbar \Delta / 2})$, with $N_{\text {MC }}$ being the total number of Monte Carlo samples. Then, we obtain the HW entropy as shown in the second line of Eq. (9).

\section{NUMERICAL PROCEDURE}

Our numerical procedure is summarized as follows:

(1) According to the initial state density matrix, an ensemble of field configurations referred to as test particles is generated.

(2) Subsequently, the classical equations of motions are solved for each test particle.
(3) The Husimi function can be estimated according to Eq. (8), and the HW entropy takes the form as given in the first line of Eq. (9).

(4) We perform the functional integral by using a Monte Carlo method as shown in the second line of Eq. (9), where $\left(\mathcal{A}_{i, m}, \mathcal{E}_{i, m}\right)$ are Gaussian random numbers.

\section{TEST IN QUANTUM MECHANICAL SYSTEM}

In the parallel test particle method, we prepare two independent sets of test particles in and out of the logarithm in Eq. (9), while they are the same samples in TP. Figure 1 shows the numerical results of the HW entropy in the twodimensional quantum mechanical system, the Hamiltonian of which is given by

$$
H=\frac{p_{1}^{2}}{2}+\frac{p_{2}^{2}}{2}+\frac{1}{2} q_{1}^{2} q_{2}^{2}+\frac{\epsilon}{4} q_{1}^{4}+\frac{\epsilon}{4} q_{2}^{4} .
$$


HIDEKAZU TSUKIJI et al.

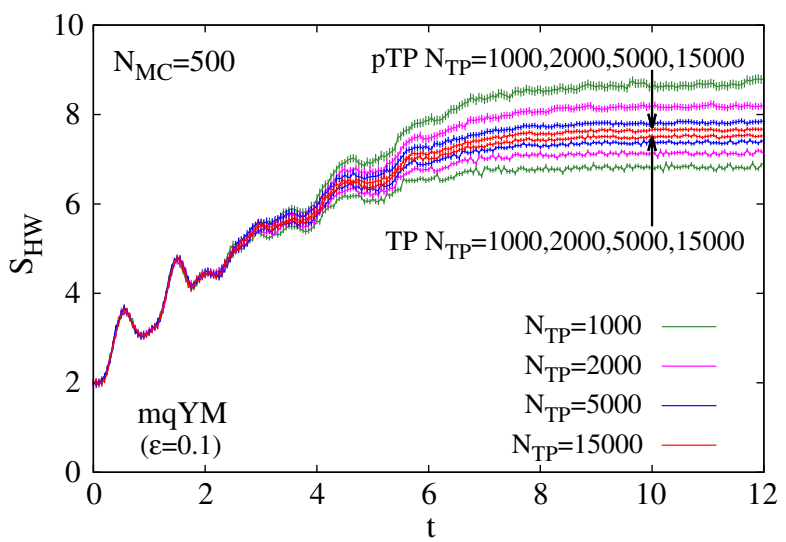

FIG. 1. Time evolution of Husimi-Wehrl entropy for the modified quantum Yang-Mills mechanics in the TP and pTP methods.

This system is called a modified quantum Yang-Mills (mqYM) model in Ref. [38]. We set the initial condition of the Wigner function as a Gaussian corresponding to the coherent state; $f_{\mathrm{W}}(p, q ; t=0)=4 \exp \left(-\omega\left(\left(p_{1}-p_{1,0}\right)^{2}+\right.\right.$ $\left.\left.\left(p_{2}-p_{2,0}\right)^{2}\right) / \hbar-\omega\left(q_{1}^{2}+q_{2}^{2}\right) / \hbar\right)$. In this calculation, we set $\hbar=\omega=\Delta=1$ and $p_{1,0}=p_{2,0}=10$. With increasing test particle number, the HW entropy is found to converge from below (above) in the TP (pTP) method, and then it is possible to give upper and lower limits of the entropy and to guess the converged value by comparing the results in the two methods.

\section{PRODUCT ANSATZ AND EXAMPLE IN A TWO-DIMENSIONAL QUANTUM MECHANICAL SYSTEM}

While the extension to the field theory on the lattice is straightforward, the DOF are so large and numerical-cost demanding in quantum field theories that we need to invoke some approximation scheme in practical calculations. We adopt the product ansatz here to avoid this difficulty.

In the ansatz, we construct the Husimi function as a product of that for 1 degree of freedom,

$$
f_{\mathrm{H}}^{(\mathrm{PA})}[A, E ; t]=\prod_{i}^{N_{D}} f_{\mathrm{H}}^{(i)}\left(A_{i}, E_{i} ; t\right),
$$

where $f_{\mathrm{H}}^{(i)}=\int \prod_{j \neq i} d A_{j} d E_{j} / 2 \pi \hbar f_{\mathrm{H}}[A, E ; t]$. By substituting this ansatz into Eq. (6), we obtain the HW entropy as a sum of the HW entropy for 1 degree of freedom,

$$
S_{\mathrm{HW}}^{(\mathrm{PA})}=-\sum_{i=1}^{N_{D}} \int \frac{d A_{i} d E_{i}}{2 \pi \hbar} f_{\mathrm{H}}^{(i)} \log f_{\mathrm{H}}^{(i)} .
$$

The entropy estimated with the product ansatz gives the upper bound of the entropy, since it holds subadditivity. The subadditivity of the entropy is expressed as
PHYSICAL REVIEW D 94, 091502(R) (2016)

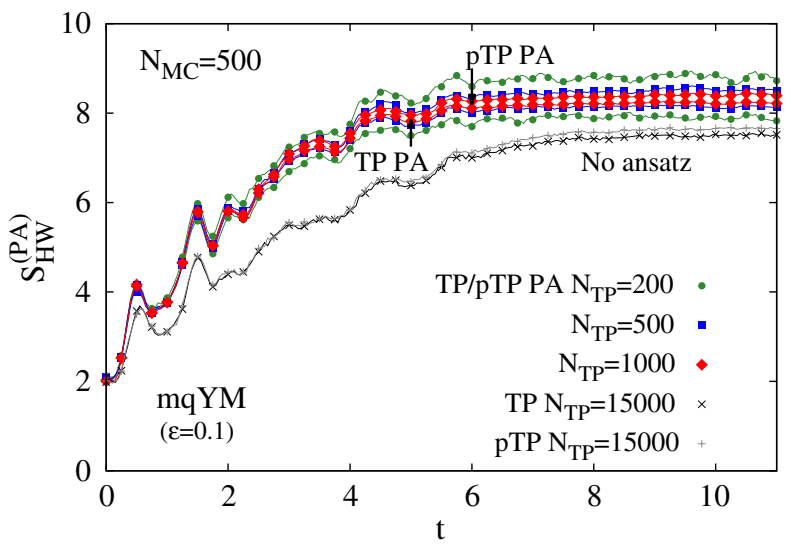

FIG. 2. The variation in the product ansatz in the twodimensional quantum model with TP and pTP methods. The green circle, blue square, and orange triangle lines are the time evolutions of HW entropy with the product ansatz in TP and pTP methods where the numbers of test particles are $N_{\mathrm{TP}}=200,500$, and 1000, respectively. The gray cross and black $x$ lines are the time evolutions of the HW entropy in both methods without the product ansatz $N_{\mathrm{TP}}=15000$.

$$
S_{1}\left(\rho_{1}\right)+S_{2}\left(\rho_{2}\right) \geq S_{12}\left(\rho_{12}\right)
$$

where $\rho_{1}=\int d \Gamma_{2} \rho_{12}$ and $\rho_{2}=\int d \Gamma_{1} \rho_{12}$ and $S_{1}$ and $S_{2}$ are subsystem entropies. In this paper, we apply it to the Husimi function and the Husimi-Wehrl entropy. Thus, the obtained entropy $S_{\mathrm{HW}}^{(\mathrm{PA})}$ gives the upper bound of $S_{\mathrm{HW}}$ due to the subadditivity;

$$
\begin{aligned}
S_{\mathrm{HW}} & \equiv S_{12 \ldots N_{D}}\left(\rho_{12 \ldots N_{D}}\right) \leq S_{1}\left(\rho_{1}\right)+S_{23 \ldots N_{D}}\left(\rho_{23 \ldots N_{D}}\right) \\
& \leq \sum_{n}^{N_{D}} S_{n}\left(\rho_{n}\right)=S_{\mathrm{HW}}^{(\mathrm{PA})} .
\end{aligned}
$$

To check the variety, we apply it to the mqYM model previously discussed. Figure 2 shows the numerical results of the HW entropy with the product ansatz $\left(S_{\mathrm{HW}}^{(\mathrm{PA})}\right)$ as well as the full entropy $\left(S_{\mathrm{HW}}\right)$, which can be found in Fig. 1. While $S_{\mathrm{HW}}^{(\mathrm{PA})}$ slightly overestimates $S_{\mathrm{HW}}$, the difference is small enough to confirm entropy production. The HW entropy with the product ansatz is found to agree with that without the ansatz within 10-20\% error in a few-dimensional quantum mechanical system. We also find that numerical results with the ansatz converge with smaller Monte Carlo samples; then, it is much more efficient from the viewpoint of numerical-cost reduction.

\section{ENTROPY PRODUCTION IN YANG-MILLS FIELD THEORY}

We apply the above-mentioned framework to the SU(2) Yang-Mills field theory. The initial condition of the Winger function is set to be a Gaussian distribution, 


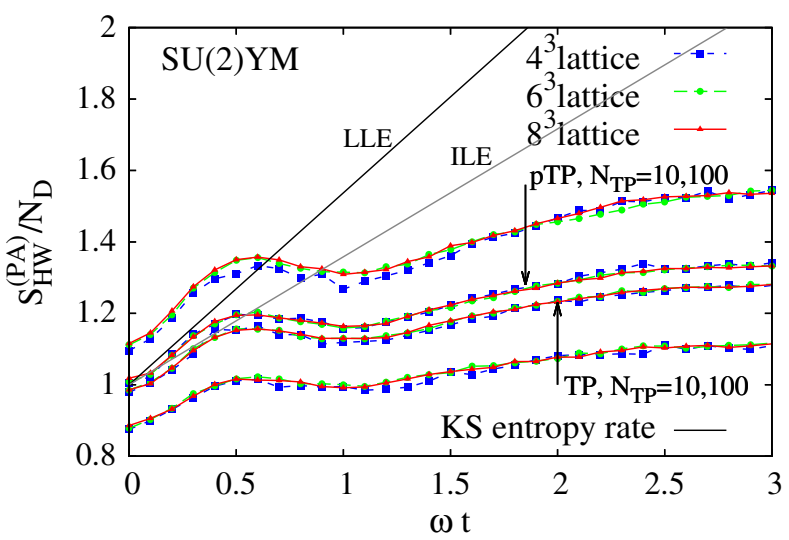

FIG. 3. The time evolution of Husimi-Wehrl entropy for the SU(2) Yang-Mills field theory on the $4^{3}, 6^{3}$, and $8^{3}$ lattices in the TP and pTP methods with the product ansatz. The blue square, green circle, and red triangle lines are the HW entropy per 1 degree of freedom on the $4^{3}$ lattice, $6^{3}$ lattice, and $8^{3}$ lattice, respectively. The black and gray solid lines show growth rates of LLE and ILE, respectively, given in Ref. [18].

$f_{\mathrm{W}}[A, E ; t=0]=2^{N_{D}} \exp \left(-\omega A^{2} / \hbar-E^{2} / \omega \hbar\right)$, which corresponds to a coherent state. The Wigner-function evolution is obtained by solving the classical EOM, and the HW entropy with the product ansatz is calculated by using the TP and pTP methods. We take the parameter set, $\hbar=\Delta / \omega=1$. Calculating the HW entropy needs $N_{\mathrm{MC}} N_{\mathrm{TP}}^{2}$ times summation as found in Eq. (9) and is numerically demanding, so we show here the results on the relatively small lattices, $4^{3}, 6^{3}$, and $8^{3}$.

In Fig. 3, we show the time evolution of the HusimiWehrl entropy per DOF with the product ansatz in the TP and pTP methods. We find that the HW entropy per DOF is independent of the lattice size, and the extensive nature of entropy is confirmed. The dependence on the number of test particle number is the same as that in quantum mechanics; with increasing Monte Carlo samples, $S_{\mathrm{HW}}^{(\mathrm{PA})}$ converges from below and above in the TP and pTP methods, respectively. The results in the TP and pTP methods approach each other with increasing $N_{\mathrm{TP}}$, and we can guess that the converged value lies between these curves. The oscillatory behavior of the HW entropy around $\omega t=0.5$ is caused by a collective motion of the phasespace distribution starting from the initial distribution that is different from the equilibrium one. After the oscillation around $\omega t=0.5$, the HW entropy increases in a monotonic way, and its growth rate decreases. This means that the collective motion in the earliest stage damps, and at later times, the entropy production rate becomes smaller, which might suggest that the system approaches a quasistationary nonequilibrium state [42]. Although this is an interesting possibility, an exploration of the possibility is beyond the scope of the present paper.

The straight lines in Fig. 3 show the KS entropy rate, which is given in Ref. [18]. The upper and lower lines show the sum of positive local and intermediate Lyapunov exponents (LLE and ILE), respectively. The LLE are obtained as the eigenvalues of the second derivative matrix of the Hamiltonian, and the ILE show the exponential growth rate in some time duration. Since the classical YM fields are conformal, the KS entropy rate should be proportional to $\varepsilon^{1 / 4}$ where $\varepsilon$ is the energy per site. The coefficients are evaluated in Ref. [18] as $R_{\mathrm{KS}}^{\mathrm{LLE}} / L^{3} \simeq 3 \times$ $\varepsilon^{1 / 4}$ and $R_{\mathrm{KS}}^{\mathrm{ILE}} / L^{3} \simeq 2 \times \varepsilon^{1 / 4}$ for the sum of positive LLE and ILE (local and intermediate KS entropy rates), respectively. These findings show that the local KS entropy rate characterizes the growth rate of the HW entropy in the early time and the intermediate KS entropy rate agrees with the average entropy growth after the initial stage.

Let us mention here the gauge dependence of the HW entropy. The present result shows the HW entropy rate is consistent with the KS entropy rate, which is shown to be gauge invariant [21]. We also note that the gauge degrees of freedom do not contribute to the chaoticity and instability $[21,23]$. For these reasons, we expect that the gauge dependence of the HW entropy production is not serious.

\section{CONCLUSION}

In summary, we have developed a novel numerical formulation and have calculated the time evolution of the Husimi-Wehrl entropy in Yang-Mills field theory in the semiclassical approximation for the first time. We have shown that the HW entropy is produced and the growth rates roughly agree with Lyapunov exponents. It should be noted that the time reversal invariance is kept in the present framework, in the time evolution of the Wigner function as well as in measuring the entropy. The produced entropy mainly comes from the complexity of the phase-space distribution.

The entropy growth will contribute to the thermalization process in relativistic heavy-ion collisions. The setup in this article is motivated by the initial stage dynamics in relativistic heavy-ion collisions. For more realistic analysis, we should choose the initial condition like the one given by the McLerran-Venugopalan model [7].

\section{ACKNOWLEDGMENTS}

This work was supported in part by the Grants-in-Aid for Scientific Research from Japan Society for the Promotion of Science (JSPS) (Grants No. 20540265, No. 23340067, No. 15K05079, and No. 15H03663), the Grants-in-Aid for Scientific Research on Innovative Areas from Ministry of Education, Culture, Sports, Science and Technology (MEXT) (Grants No. 23105713, No. 24105001, and No. 24105008), and by the Yukawa International Program for Quark-Hadron Sciences. T. K. is supported by the Core Stage Back Up program in Kyoto University. 


\section{HIDEKAZU TSUKIJI et al.}

[1] J. von Neumann, Nachrichten von der Gesellschaft der Wissenschaften zu G̈ottingen, Mathematisch-Physikalische Klasse 1927, 273 (1927); Mathematische Grundlagen der Quantenmechanik (Springer, Berlin, 1932).

[2] W. H. Zurek, Phys. Today 44, 36 (1991).

[3] J. von Neumann, Z. Phys. 57, 30 (1929).

[4] S. Goldstein, J. L. Lebowitz, R. Tumulka, and N. Zanghi, Eur. Phys. J. H 35, 173 (2010).

[5] H. Tasaki, arXiv:1507.06479.

[6] S. Ryu and T. Takayanagi, Phys. Rev. Lett. 96181602 (2006).

[7] L. D. McLerran and R. Venugopalan, Phys. Rev. D 492233 (1994); 49, 3352 (1994); 50, 2225 (1994).

[8] U. Heinz and P. Kolb, Nucl. Phys. A702 269 (2002).

[9] R. Baier, A. H. Mueller, D. Schiff, and D. T. Son, Phys. Lett. B 502, 51 (2001).

[10] P. Romatschke and R. Venugopalan, Phys. Rev. Lett. 96, 062302 (2006).

[11] B. Muller and A. Schafer, Phys. Rev. C 73, 054905 (2006).

[12] J. Berges, S. Scheffler, and D. Sexty, Phys. Rev. D 77, 034504 (2008).

[13] A. Iwazaki, Phys. Rev. C 77, 034907 (2008).

[14] H. Fujii and K. Itakura, Nucl. Phys. A809, 88 (2008).

[15] R. J. Fries, B. Muller, and A. Schafer, Phys. Rev. C 78, 034913 (2008).

[16] R. J. Fries, B. Muller, and A. Schafer, Phys. Rev. C 79, 034904 (2009).

[17] H. Fujii, K. Itakura, and A. Iwasaki, Nucl. Phys. A828, 178 (2009).

[18] T. Kunihiro, B. Muller, A. Ohnishi, A. Schafer, T. T. Takahashi, and A. Yamamoto, Phys. Rev. D 82, 114015 (2010).

[19] K. Fukushima and F. Gelis, Nucl. Phys. A874, 108 (2012).

[20] T. Epelbaum and F. Gelis, Phys. Rev. Lett. 111, 232301 (2013).
PHYSICAL REVIEW D 94, 091502(R) (2016)

[21] H. Iida, T. Kunihiro, B. Muller, A. Ohnishi, A. Schafer, and T. T. Takahashi, Phys. Rev. D 88, 094006 (2013).

[22] H. Iida, T. Kunihiro, A. Ohnishi, and T. T. Takahashi, arXiv: 1410.7309 .

[23] S. Tsutsui, H. Iida, T. Kunihiro, and A. Ohnishi, Phys. Rev. D 91, 076003 (2015).

[24] S. Tsutsui, T. Kunihiro, and A. Ohnishi, Phys. Rev. D 94, 016001 (2016).

[25] M. Ruggieri, A. Puglisi, L. Oliva, S. Plumari, F. Scardina, and V. Greco, Phys. Rev. C 92, 064904 (2015).

[26] I. Arsenej et al., Nucl. Phys. A757, 1 (2005).

[27] K. Adcox et al., Nucl. Phys. A757, 184 (2005).

[28] B. B. Back et al., Nucl. Phys. A757, 28 (2005).

[29] J. Adam et al., Nucl. Phys. A757, 102 (2005).

[30] B. Muller, J. Schukraft, and B. Wyslouch, Annu. Rev. Nucl. Part. Sci. 62, 361 (2012).

[31] A. J. Lichtenberg and M. A. Lieberman, Regular and Chaotic Dynamics (Springer, New York, 1992).

[32] Y. B. Pesin, Russ. Math. Surv. 32, 55 (1977).

[33] V. Latora and M. Baranger, Phys. Rev. Lett. 82, 520 (1999).

[34] E. P. Wigner, Phys. Rev. 40, 749 (1932).

[35] K. Husimi, Proc. Phys. Math. Soc. Jpn. 22, 264 (1940).

[36] A. Wehrl, Rev. Mod. Phys. 50, 221 (1978).

[37] T. Kunihiro, B. Muller, A. Ohnishi, and A. Schafer, Prog. Theor. Phys. 121, 555 (2009).

[38] H. Tsukiji, H. Iida, T. Kunihiro, A. Ohnishi, and T. T. Takahashi, Prog. Theor. Exp. Phys. 2015, 083A01 (2015)

[39] T. Lappi and L. McLerran, Nucl. Phys. A772 200 (2006).

[40] S. Mrowczynski and B. Muller, Phys. Rev. D 50, 7542 (1994).

[41] A. Polkovnikov, Ann. Phys. (Amsterdam) 325, 1790 (2010).

[42] J. Berges, K. Boguslavski, S. Schlichting, and R. Venugopalan, Phys. Rev. D 89, 074011 (2014); 89, 114007 (2014). 